\title{
Role of Stable Modes in the ITG-Driven Instability in a Mode-Coupled System
}

\author{
Paul P. HILSCHER ${ }^{1)}$, Kenji IMADERA ${ }^{1)}$, Jiquan LI $^{1)}$ and Yasuaki KISHIMOTO ${ }^{1,2)}$ \\ ${ }^{1)}$ Graduate School of Energy Science, Kyoto University, Gokasho, Uji, Kyoto 611-0011, Japan \\ ${ }^{2)}$ Institute of Advanced Energy, Kyoto University, Gokasho, Uji, Kyoto 611-0011, Japan
}

(Received 10 June 2013 / Accepted 19 August 2013)

\begin{abstract}
Kinetic damping in linear gyrokinetic (GK) Vlasov simulations is found to exhibit a bifurcation at the collisionality $\beta_{c}=\beta_{c}^{\star}$, above which, i.e. $\beta_{c}>\beta_{c}^{\star}$, the damping is represented by a Landau eigenmode in velocity space, while below which, i.e. $\beta_{c}<\beta_{c}^{\star}$, by the phase mixing of a finite number of marginally stable, discretized Case-van Kampen eigenmodes. The latter causes a recurrence that restricts the damping and then the energy transfer from wave to particles within a finite recurrence time. In order to address whether the stabilization effect due to such stable damped modes on unstable modes via mode coupling can be evaluated in long timescale GK simulations, we introduced a triad model consisting of stable and unstable modes incorporated with a tertiary vortex flow. We identified $\beta_{c}^{\star}$ numerically and found that the stabilization effect works properly beyond the recurrence time even in the phase mixing regime across $\beta_{c}=\beta_{c}^{\star}$.
\end{abstract}

(c) 2013 The Japan Society of Plasma Science and Nuclear Fusion Research

Keywords: gyrokinetics, Landau damping, Case-van Kampen eigenmode, mode coupling

DOI: $10.1585 /$ pfr.8.1303151

In plasma turbulence, stable modes play an important role in absorbing fluctuating free energy as a sink, which leads to saturation. Such a phenomenon commonly exists in various linear and nonlinear mode coupling processes. For example, nonlinear energy cascades occur through a three-wave coupling in drift wave turbulence. On the other hand, the unstable ion temperature gradient (ITG) mode is suppressed by the linear mode coupling produced by an externally imposed static vortex flow or magnetic island, forming a global ITG structure with the same growth rates and frequencies [1]. Mode coupling mediates the underlying suppression mechanisms of unstable modes by the dissipation effect of stable modes. Such stable modes originate from various damping processes. They are characterized by collisional (viscous) damping in a fluid model while they result from Landau damping and the so-called finite Larmor radius (FLR) effects in the kinetic system. Hence, Landau damping is crucial in energy transfer processes because it predominantly affects the fluctuation dynamics in both linear and nonlinear regimes.

However, in collisionless gyrokinetic (GK) simulations, Landau damping exhibits different time evolutions depending on the choice of the initial distribution in velocity space due to the appearance of marginally stable Case-van Kampen ( $\mathrm{CvK})$ eigenmodes in the discretized velocity space. Namely, we observe a marginal behavior with neither growth nor damping for a random noise distribution. On the other hand, a damping accompanied by the recurrence phenomenon occurs for the Maxwellian distribution [2], where damping is terminated within a finite time determined by the mesh size $v$. Consequently, the damping rate averaged over a timescale longer than the recurrence time becomes zero. Such damping with recurrence has been found to result not from Landau damping as a discrete eigenmode but from the phase mixing of a finite number of $\mathrm{CvK}$ eigenmodes. This is in contrast to the unstable ITG mode, which is always found to be an eigenmode of the GK Vlasov-Poisson (VP) system. These issues have been intensively studied among researchers in the field [2-9].

To determine Landau damping through an eigenmode of the VP system, here referred to as Landau eigenmode, a finite collisionality $\beta_{c}$ larger than the critical value $\beta_{c}^{\star}$, i.e. $\beta_{c}>\beta_{c}^{\star}$, is necessary which leads to the damping for the $\mathrm{CvK}$ eigenmodes below the growth rate that is predicted by the Landau theory so that a Landau eigenmode appears. In other words, a bifurcation of the solution for the stable mode in a discretized VP system occurs at $\beta_{c}=\beta_{c}^{\star}$. Once the Landau eigenmode is revealed, the recurrence phenomenon disappears and the damping is found to be independent of the initial perturbation, e.g., random noise and Maxwellian distribution. In general, a higher (lower) collisionality is required for a lower (higher) resolution to damp the CvK eigenmodes [3].

Now, the question arises of whether the stabilization effect of unstable modes through stable modes is correctly evaluated beyond the recurrence time, in collisionless nonlinear GK simulations or in a linearly coupled VlasovPoisson system, where the linear coupling between un- 
stable and stable modes arises through an external vortex flow [1]. To address this problem, we investigated the characteristics of stable ITG modes in sheared slab geometry by incorporating two approaches: GK Vlasov simulations as an initial value problem (IVP) and eigenvalue analysis, both of which are equally discretized in velocity space. The imposed vortex flow, which induces the linear coupling for different poloidal modes, is considered to originate from a tearing mode and is given by $\phi_{\mathrm{VF}}=$ $\epsilon \tanh (x) \exp \left[-(x / \epsilon)^{2}\right] \sin \left(k_{\mathrm{VF}} y\right)$, where $k_{\mathrm{VF}}=2 \pi / L_{y}, L_{y}=$ 64 and $\epsilon$ represent the wavenumber and the amplitude, respectively. The linearized GK equations with conventional normalization [10] for the drift waves is given by

$$
\frac{\partial f_{1, \vec{k}_{\perp}}}{\partial t}=\mathcal{S}_{\vec{k}_{\perp}}+\mathcal{L}_{\vec{k}_{\perp}}+\mathcal{D}_{\mathrm{LB}, \vec{k}_{\perp}}+\left[\phi_{\mathrm{VF}}, f_{1, \vec{k}_{\perp}}\right],
$$

where $f_{1}$ is the perturbed distribution function from the Maxwellian background and $\mathcal{S}_{\vec{k}_{\perp}}$ is the source term that depends on $\eta_{i}=L_{n} / L_{T}$, i.e., the ratio of temperature gradient scale length to density gradient scale length. Furthermore, $\mathcal{L}_{\vec{k}_{\perp}}$ consists of the parallel convection and the linear Landau damping term, and $\mathcal{D}_{\mathrm{LB}, \vec{k}_{\perp}}$ is the LenardBernstein collisional operator. The last term is the Poisson bracket which induces linear mode coupling through the vortex flow.

Figure 1 shows the results of directly solving Eq. (1) as an IVP. Note that the long timescale average exceeding the recurrence time leads to an almost zero damping rate for the stable modes independent on the initial condition. Here we illustrate two cases: without $(\epsilon=0)$ and with $(\epsilon=0.4)$ coupling by the vortex flow. Each case is considered both without $\left(\beta_{c}=0\right)$ and with $\left(\beta_{c}=2 \times 10^{-3}\right)$ collision, where $\beta_{c}>\beta_{c}^{\star} \sim 10^{-3}$ so that we resolve the

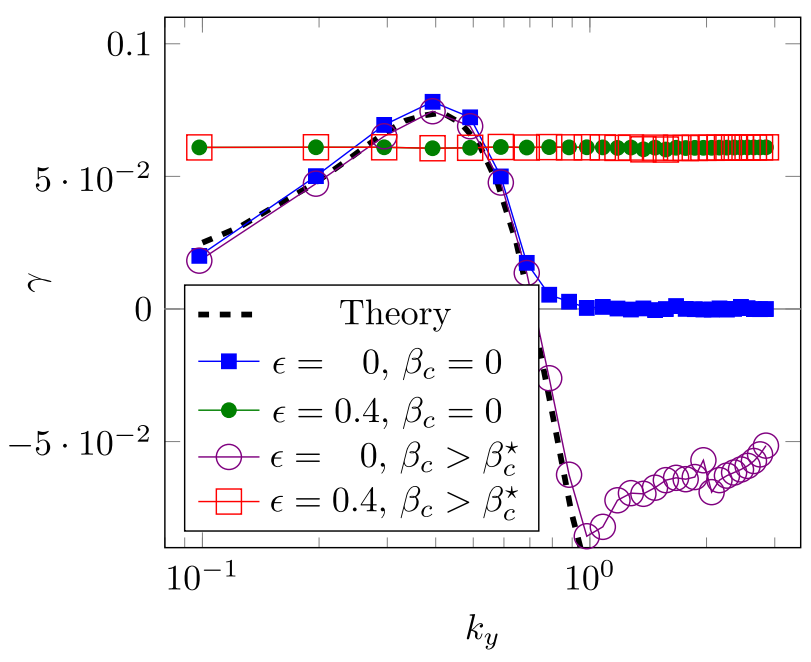

Fig. 1 Growth rates of the electrostatic potential from the GK Vlasov simulation as an IVP in a coupled system $(\epsilon=$ $0.4)$ mediated by a vortex flow and in an uncoupled system $(\epsilon=0)$. The dashed curve corresponds to the theoretical solution of the uncoupled system $\left(\hat{s}=0.6, \eta_{i}=6\right)$.
Landau eigenmodes for $k_{y}<1$. The result of solving the theoretical dispersion relation (without coupling and collisions) is also shown by the dashed line. In the absence of coupling, the unstable ITG modes show almost the same results without sensitively depending on $\beta_{c}$, indicating that the selected collisionality is sufficiently small while stable modes show a difference depending on $\beta_{c}$. Namely, stable modes show marginal behavior for $\beta_{c}=0$, whereas they show damping behavior with nearly the same growth rate as predicted by Landau theory for $\beta_{c}=2 \times 10^{-3}$. As discussed above, this is expected, because the former results from the phase mixing of the associated $\mathrm{CvK}$ eigenmodes, while the latter results from the appearance of the Landau eigenmode in case the $\mathrm{CvK}$ eigenmodes are sufficiently damped. On the other hand, for the case with a vortex flow, i.e., $\epsilon=0.4$, IVP simulations show that the vortex flow stabilizes the ITG mode by coupling the unstable and stable components, leading to the formation of a global structure, where all poloidal modes have the same growth rates as shown in Fig. 1. These results are consistent with those obtained from gyrofluid simulations [1]. However, it is interesting to note that the cases with and without collisions reveal similar results regardless of whether Landau damping in the absence of coupling (which is considered to be the origin of the stabilization in the presence of coupling), arises from phase mixing $\left(\beta_{c}<\beta_{c}^{\star}\right)$ or from a Landau eigenmode $\left(\beta_{c} \geq \beta_{c}^{\star}\right)$, as discussed in Fig. 1. Therefore, it is worthwhile to investigate whether the stable modes play a role as an energy transfer channel for unstable modes in the collisionless limit.

To answer this question, we propose a reduced model that only involves coupling of three modes of the electrostatic potential, i.e., an unstable mode, a stable mode, and an externally imposed tertiary vortex flow. In this minimal model, magnetic shear is ignored and the FLR effect up to the first order is considered. The equilibrium magnetic field is given by $\vec{B}_{0}=B_{0}(\hat{z}+\theta \hat{y})$ with $\theta \ll 1$. Note that for a fixed $k_{x}$, we can calculate the dispersion relation, where the velocity integral is expressed using the Fred-Conte plasma dispersion function [11]. Here, we set $k_{x}=0$, which gives the largest growth rate. The Taylor expansion around $x=0$ has been applied to the vortex flow to more clearly represent the leading tearing parity term which results in $\phi_{\mathrm{VF}}=-\frac{1}{2} i x \epsilon\left(\exp \left[i k_{\mathrm{VF}} y\right]-\exp \left[-i k_{\mathrm{VF}} y\right]\right)$. The inclusion of the vortex flow leads to a coupling of neighboring modes as $k_{y}^{\prime} \pm k_{\mathrm{VF}}$. Here, we only consider a coupling pair given by $k_{y}^{\prime}=1.3$ and $k_{y}^{\prime \prime}=k_{y}^{\prime}+k_{\mathrm{VF}}=1.8$ and neglect the other as the dynamic is qualitatively equal. The resulting equations for the unstable mode $k_{y}^{\prime}$ and the stable mode $k_{y}^{\prime \prime}$ are given by

$$
\begin{aligned}
& \frac{\partial f_{1, k_{y}^{\prime}}}{\partial t}=S_{k_{y}^{\prime}}+L_{k_{y}^{\prime}}+\mathcal{D}_{\mathrm{LB}, k_{y}^{\prime}}+C_{k_{y}^{\prime \prime}}, \\
& \frac{\partial f_{1, k_{y}^{\prime \prime}}}{\partial t}=S_{k_{y}^{\prime \prime}}+L_{k_{y}^{\prime \prime}}+\mathcal{D}_{\mathrm{LB}, k_{y}^{\prime \prime}}+C_{k_{y}^{\prime}},
\end{aligned}
$$

where 

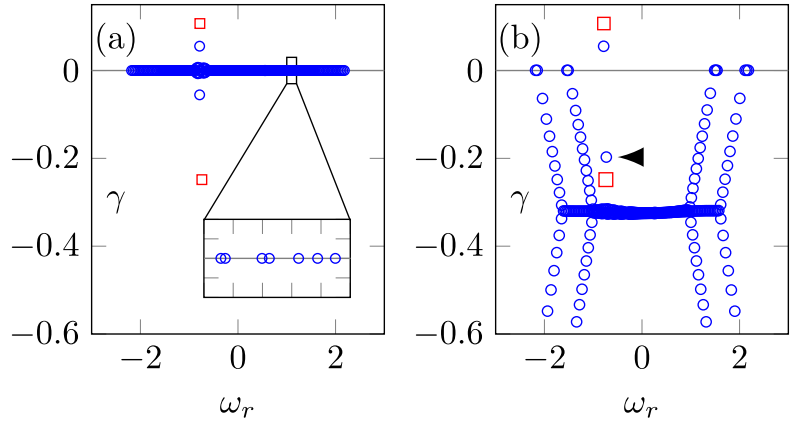

Fig. 2 Eigenvalue spectra (०) of the coupled system with $\epsilon=0.1$ for the collisionless case (a) with $\beta_{c}=0$, and collisional case (b) with $\beta_{c}=5 \times 10^{-4}$ are shown together with the Landau solution of the uncoupled case ( $\square$ ).

$$
C_{k_{y}^{\prime}}=+\frac{i \epsilon}{2} k_{y}^{\prime} f_{1, k_{y}^{\prime}} \quad \text { and } \quad C_{k_{y}^{\prime \prime}}=-\frac{i \epsilon}{2} k_{y}^{\prime \prime} f_{1, k_{y}^{\prime \prime}},
$$

are the cross-coupling terms from the Poisson bracket in Eq. (1).

On solving the discretized Eq. (2a) and (2b), we reformulate them as an eigenvalue problem by assuming a harmonic time dependence for $f_{1, \vec{k}_{\perp}}$ as $\tilde{f}_{1, \vec{k}_{\perp}} \mathrm{e}^{-i \omega t}$. Thus, we get

$$
-i \omega \tilde{f}_{1, \vec{k}_{\perp}}=\mathcal{G}_{k_{y}} \cdot \tilde{f}_{1, \vec{k}_{\perp}},
$$

where $\mathcal{G}_{k_{y}}$ is the so-called linear GK operator. The explicit form using $\tilde{f}_{1, k_{y}}=\left(f_{1, k_{y}^{\prime}}, f_{1, k_{y}^{\prime \prime}}\right)$ is then given by

$$
-i \omega\left(\begin{array}{l}
f_{1, k_{y}^{\prime}} \\
f_{1, k_{y}^{\prime \prime}}
\end{array}\right)=\underbrace{\left(\begin{array}{cc}
\mathcal{G}_{k_{y}^{\prime}} & \mathcal{C}_{k_{y}^{\prime \prime}} \\
\mathcal{C}_{k_{y}^{\prime}} & \mathcal{G}_{k_{y}^{\prime \prime}}
\end{array}\right)}_{\mathcal{G}_{k_{y}^{\prime}, k_{y}^{\prime \prime}}}\left(\begin{array}{c}
f_{1, k_{y}^{\prime}} \\
f_{1, k_{y}^{\prime \prime}}
\end{array}\right),
$$

where the off-diagonal elements, i.e. $C_{k_{y}^{\prime}}$ and $C_{k_{y}^{\prime \prime}}$, lead to the mode coupling. Note that the eigenvectors of the operator $\mathcal{G}_{k_{y}^{\prime}, k_{y}^{\prime \prime}}$ are twice the size of those for the operator $\mathcal{G}_{k_{y}}$ in Eq. (4). Here an equidistant discretization in velocity space is used with $L_{v}= \pm 4$ and a grid number of $N_{v}=128$. Physical parameters are set to $\eta_{i}=6, \theta=0.3, \epsilon=0.1$. The eigenvalue problem is solved by using the KrylovSchur iteration procedure, for example, as provided by SLEPc [12].

Figure 2 shows the eigenvalues (circles) in (a) the collisionless and (b) collisional cases $\left(\beta_{c}=10^{-3}\right)$, respectively, including the analytical solution from the dispersion relation in the uncoupled system (squares). In Fig. 2(a), an unstable global mode with its complex conjugate (time reversed) solution is observed; others correspond to the marginally stable CvK eigenmodes. By comparing with the analytical solution of the uncoupled case, we find that the unstable global mode is stabilized through coupling even though no stable eigenmode exists. In presence of collision larger than the critical value, i.e., $\beta_{c}>\beta_{c}^{\star} \sim 5 \times 10^{-4}$, the collision strongly damps the CvK eigenmodes, resulting in the stable global mode, shown by the arrow in Fig. 2(b), appearing as an eigenmode in velocity space. We find that the growth rate of the unstable
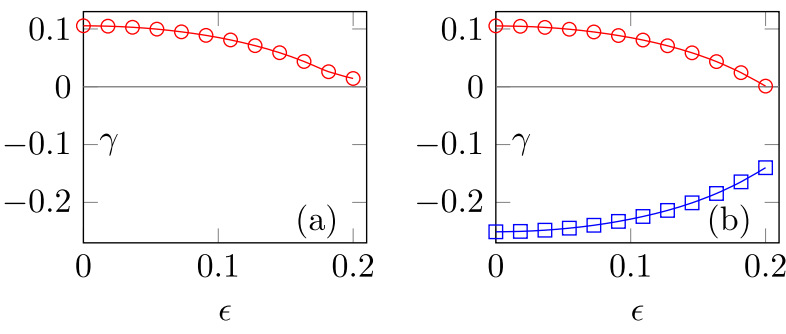

Fig. 3 Growth rates of the unstable $(\ominus) / \operatorname{stable}(\boxminus)$ global eigenmodes versus the coupling parameter $\epsilon$ for $\beta_{c}=0$ (a) and $\beta_{c}=5 \times 10^{-4}(\mathrm{~b})$ in the coupled system.

global mode, which is considered to be stabilized by the eigenmode, is the same as that in Fig. 2(a), i.e., in the collisionless case. In contrast to the case without coupling, the damping rate of the Landau damped eigenmode is reduced, i.e., destabilization occurs. The result is consistent with the concept that the energy of the unstable mode is partially transferred to the stable mode which maintains the energy conservation. From these results, we conclude that mode coupling between unstable and stable modes through a tertiary mode and the resultant energy transfer can be properly reproduced so that the stable mode, which is represented by the phase mixing of the $\mathrm{CvK}$ eigenmodes, persist.

To further test this revealing insight, a parametric scan over the coupling parameter $\epsilon$ is performed as shown in Fig. 3. In the collisional case, the growth rate of the stable global mode increases with increasing $\epsilon$, whereas the unstable global mode is stabilized with almost the same growth rate, similar to that in the collisionless case. A stable global mode is not represented as an eigenmode in velocity space in the absence of collision, hence their growth rates are not shown in Fig. 3(a). These results show that the linear stabilization mechanism due to mode coupling is independent of collision and thus the existence of the Landau damped mode as a true eigenmode of the VP system is not essential.

In conclusion, stable modes play an important role in suppressing instability through mode coupling in GK Vlasov simulations. This stabilization effect is independent of whether the energy transfer results from phase mixing of the CvK eigenmodes or a true Landau eigenmode.

[1] Z.X. Wang et al., Phys. Rev. Lett. 103(1), 015004 (2009).

[2] J. Canosa et al., J. Comp. Phys. 15(1), 34 (1974).

[3] P.P. Hilscher et al., Phys. Plasmas 20(8), 082127 (2013).

[4] F.C. Grant and M.R. Feix, Phys. Fluids 10(4), 696 (1967).

[5] N.G. van Kampen, Physica 21(6-10), 949 (1955).

[6] K.M. Case, Ann. Phys. 7(3), 349 (1959).

[7] C.S. Ng et al., Phys. Rev. Lett. 83(10), 1974 (1999).

[8] V. Bratanov et al., Phys. Plasmas 20(2), 022108 (2013).

[9] H.S. Xie, arXiv, 1305.6476 (2013).

[10] P.P. Hilscher et al., Plasma Fusion Res. 8, 2403040 (2013).

[11] M. Nakata et al., Phys. Plasmas 18(1), 012303 (2011).

[12] V. Hernandez et al., ACM Trans. Math. Software 310(3), 351 (2005). 